\title{
Megakaryocyte Differentiation
}

National Cancer Institute

\section{Source}

National Cancer Institute. Megakaryocyte Differentiation. NCI Thesaurus. Code C18013.

A biological process that refers to the maturation of megakaryocytes and the release of platelets. 